Sir,

\section{Bilateral retrobulbar neuritis following measles in an adult}

Optic neuritis associated with viral infections has been well reported but infrequently. It has been seen particularly during or after an attack of childhood measles, associated mostly with encephalomyelitis. We present a woman with measles infection and visual loss resulting from retrobulbar optic neuritis. The present case is of particular interest because of the optic nerve involvement following measles in an adult without encephalomyelitis.

\section{Case report}

A 25-year-old woman was referred to us with bilateral loss of vision 11 days after the onset of a measles rash. Her child had suffered from measles 3 weeks earlier. On examination, the lids and eyeballs appeared normal. The pupils reacted sluggishly to light. The visual acuities of both eyes were limited to counting fingers at $2 \mathrm{~m}$.

Biomicroscopic examination of the conjunctiva, cornea, anterior chamber, iris and lens as well as the fundus revealed no abnormality. Intraocular pressures were normal. There were large central scotomata bilaterally, but the optic discs were normal on ophthalmoscopy (Fig. 1). Examination also revealed dyschromatopsia.

The patient's medical and surgical history were unremarkable. There was no history of multiple sclerosis or other ocular or central nervous system disease. The patient had no known allergies and was not taking medication. She had no past history of measles. Complete physical examination including the central and peripheral nervous system revealed no abnormality. Other possible infectious causes of rashes with neurological dysfunction such as Epstein-Barr virus infection were excluded by clinical examination and laboratory investigations.

The results of computed tomography and magnetic resonance imaging of the head and orbits were normal. The electroencephalogram demonstrated no electrical evidence of an encephalitic process. The cerebrospinal fluid contained no white cells and protein and glucose levels were normal. IgG content of the total cerebrospinal fluid protein was increased, but oligoclonal bands were absent from the cerebrospinal fluid. Serological test for syphilis was negative. Serum measles antibody titres were $1 / 256$ on admission, declining to $1 / 16$ a month later, confirming a recent measles infection. Visual evoked potentials measured during the first week of admission showed a greatly diminished response bilaterally.

The patient was treated with intravenous methylprednisolone, $250 \mathrm{mg}$ every $6 \mathrm{~h}$ for 3 days, followed by $60 \mathrm{mg} /$ day oral prednisone. The daily dose of prednisone was reduced by $10 \mathrm{mg}$ each week to zero. Six weeks later, visual acuities were $20 / 100$ in the right eye and 20/200 in the left eye. Eight months later the visual acuity of the right eye was $20 / 20$ and that of the left eye was $20 / 25$. Visual evoked potentials revealed no prolonged P100 latency bilaterally, but abnormal amplitudes were still recorded, especially in the left eye (Fig. 2).

\section{Comment}

Apart from conjunctival and corneal signs, other ocular manifestations of measles are seen infrequently. ${ }^{1,2}$ Optic neuritis is a rare ocular complication of measles that is seen in childhood measles as an expression of encephalomyelitis. The optic neuritis usually occurs about 1 week after the onset of initial symptoms. The optic nerve generally presents as bilateral papillitis with a swollen nerve head. The visual prognosis is usually good after the infection clears. ${ }^{3-7}$ However, to the best of our knowledge there is no report of a well-studied case of bilateral retrobulbar neuritis as a complication of measles in an adult with no other symptoms and signs of encephalitis.

The pathogenesis by which a viral illness leads to subsequent optic neuropathy is controversial. As optic neuritis may sometimes occur without associated neurological signs, the term localised encephalitis has been considered for the process. ${ }^{8}$ In our case, the severe visual loss with marked improvement, the delay after the initial infection and bilateral simultaneous optic nerve

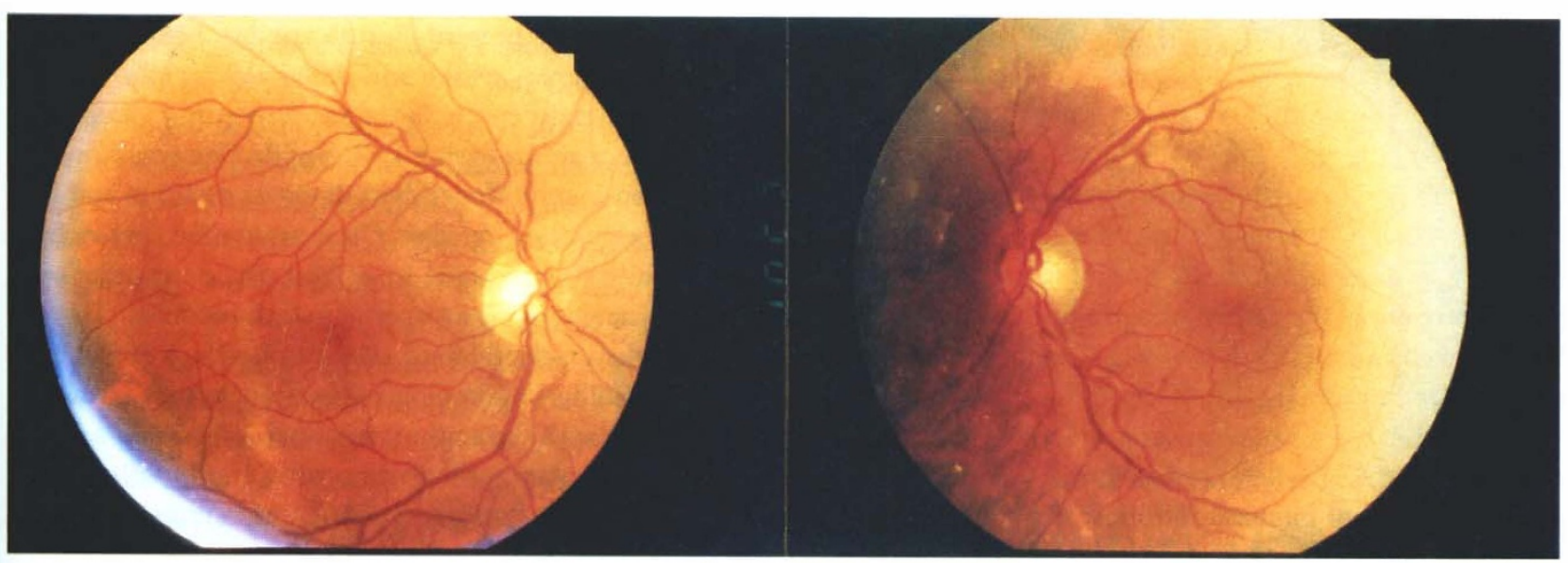

Fig. 1. Bilateral normal optic discs and fundus appearance. 


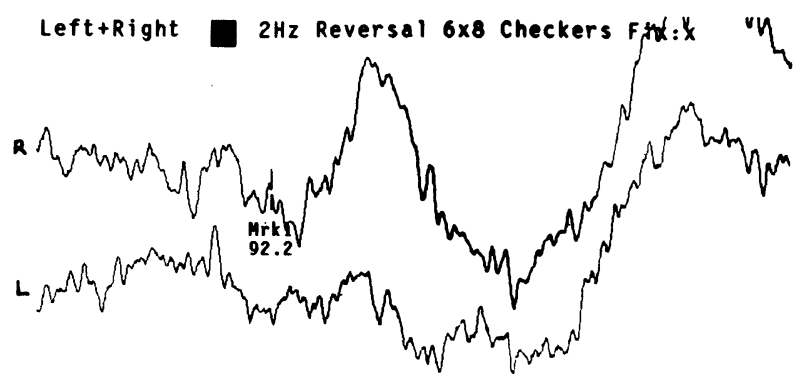

Fig. 2. Pattern-reversal visual evoked potentials show normal P100 latencies but diminished amplitudes in the right eye (upper tracing) and especially in the left eye (lower tracing).

involvement are strong support for a generalised mechanism of injury. This possibility resulted from a systemic autoimmune demyelination, rather than random, viral invasion of both optic nerves. In the present case, the interval of 11 days between the onset of the measles rash and the development of bilateral visual loss is enough for host immune mechanisms to become active. Possible mechanisms for the secondary autoimmune process include molecular mimicry between viral and neural antigens, or incorporation of virally coded antigens into neural tissue such as oligodendrocyte membrane or myelin sheath. Another factor may be inherited susceptibility to the production of an autoimmune response. All possible mechanisms are likely to result in demyelination. Recovery of good vision may also be consistent with primary demyelination. However, why the optic nerves were singled out in our patient remains unclear.

Visual evoked potentials 8 months later revealed low amplitudes but normal latency bilaterally. This is because some optic nerve axons might have been structurally disrupted as a sequel of acute inflammation.

It is not suggested that the present case is a variant of multiple sclerosis. Studies of simultaneous bilateral optic neuritis in adults have shown that subsequent development of multiple sclerosis is uncommon. ${ }^{9}$ Moreover, our patient had neither a family history nor a previous or subsequent attack to suggest multiple sclerosis and oligoclonal bands were absent from her cerebrospinal fluid.

The visual improvement following high-dose corticosteroid therapy was good in our patient, although spontaneous recovery without treatment has been reported. ${ }^{10,11}$ The natural course of acute bilateral optic neuritis following viral infections tends to be rather benign, with good prognosis for return of vision to nearnormal levels. There are also no controlled studies convincingly demonstrating that any form of therapy significantly alters the course of optic neuritis. However, corticosteroids are of theoretical value in suppressing the presumptive immunological reaction and thus may shorten the recovery time. ${ }^{12}$ Therefore, unless contraindicated, high-dose corticosteroid therapy may be justified in cases of bilateral retrobulbar optic neuritis following viral infections such as measles, or when one eye has previously defective vision.

\section{References}

1. Dekkers NWHM. Rubeola. In: Fraunfelder FT, Roy FH editors. Current ocular therapy, 4th ed. Philadelphia: WB Saunders, 1995:110.

2. Metz HS, Harkey ME. Pigmentary retinopathy following maternal measles (morbilli) infection. Am J Ophthalmol 1968;66:1107-10.

3. Heath P. Measles encephalitis: a clinical report of some eye findings. Am J Ophthalmol 1932;15:130-4.

4. Jennings GH. Loss and recovery of sight in measles encephalitis. Lancet 1952;II:66-7.

5. Bedrossian RH. Neuroretinitis following measles. J Pediatr 1955;46:329-31.

6. Tyler HR. Neurological complications of rubeola (measles). Medicine 1957;36:147-67.

7. Srivastava SP, Nema HV. Optic neuritis in measles. Br J Ophthalmol 1963;47:180-1.

8. Copenhaver RM. Chickenpox with retinopathy. Arch Ophthalmol 1966;75:199-200.

9. Parkin PJ, Hierons R, McDonald WI. Bilateral optic neuritis: a long term follow up. Brain 1983;107:951-64.

10. Hatch HA. Bilateral optic neuritis following chickenpox. J Pediatr 1949;34:758-9.

11. Meadows SP. Retrobulbar and optic neuritis in childhood and adolescence. Trans Ophthalmol Soc UK 1969;89:603-38.

12. Compston A. Methylprednisolone and multiple sclerosis. Arch Neurol 1988;45:669-75.

Yüksel Totan, MD

Osman Çekiç, MD

Department of Ophthalmology

Inönü University Medical Faculty

Turgut Özal Medical Center

Malatya, Turkey

Yüksel Totan, MD

Melike Sokak, No. 11/11

Etlik, Ankara,

Turkey

Tel: +903123212783

e-mail: ytotan@hotmail.com

Sir,

Idiopathic hypertrophic chronic pachymeningitis presenting with acute visual loss

\section{Clinical details}

Three days before presentation to another hospital, a 56-year-old woman awoke and noted almost total loss of vision in her right eye without any preceding visual disturbance, pain or systemic ill-health. She was known to have non-insulin-dependent diabetes mellitus and to smoke 20 cigarettes per day. On examination, her right visual acuity was reduced to, at best, the perception of hand movements in the superior nasal quadrant. There was a marked right relative afferent pupillary defect but otherwise full ophthalmological examination of both eyes was normal. General examination and her plasma viscosity were also normal and a presumptive diagnosis of non-arteritic posterior ischaemic optic neuropathy (PION) was made and conservative management instituted. 